\title{
Van der Meer calibration of the CMS luminosity detectors in 2017
}

\author{
Paul Lujan on behalf of the CMS collaboration* \\ University of Padova/INFN \\ E-mail: paul.lujan@cern.ch
}

Precision measurement of luminosity delivered by the LHC to the CMS experiment is a vital component of many physics analyses and important for optimal machine performance. In Run 2, the luminosity at CMS is measured by three online luminometers, the Pixel Luminosity Telescope (PLT), Fast Beam Conditions Monitor (BCM1F), and the hadronic forward calorimeter $(\mathrm{HF})$, which provide high-precision real-time luminosity measurements. They are complemented by two offline measurements, one using pixel cluster counting (PCC) in the main CMS pixel detector, and one using the rate of muon stubs in the drift tubes (DT). In order to calibrate the luminosity measurements, a series of van der Meer (VdM) scans is performed, in which the absolute luminosity can be calculated to determine a calibration. In the VdM scans, the separation of the beam is varied and the resulting profile of luminosity as a function of separation is fitted in order to determine the beam overlap width, from which the overall luminosity can be calculated. This analysis presents the results of this calibration, as well as the various systematic effects taken into account in the measurement.

The 39th International Conference on High Energy Physics (ICHEP2018)

4-11 July, 2018

Seoul, Korea

${ }^{*}$ Speaker. 
At the CMS experiment at the CERN LHC [1], precision measurement of the luminosity is important both in real time in order to monitor and optimize machine performance, as well as offline in order to provide the best possible inputs for physics analyses. The CMS luminosity measurement is performed by a total of five detectors; each system measures a certain rate (rate of hits, coincidences, clusters, etc.) which is related to the overall instantaneous luminosity $L_{\text {inst }}$ via the visible cross-section $\sigma_{\text {vis }}: R=\sigma_{\text {vis }} L_{\text {inst }}$. To determine this calibration constant, a set of special scans, called van der Meer (VdM) scans after their inventor, is performed.

The VdM scan methodology uses the fact that the luminosity can be expressed as a function of the proton density functions $\rho_{i}$ for the two beams, $L_{\text {inst }}=N_{1} N_{2} \int \rho_{1} \rho_{2} \mathrm{~d} x \mathrm{~d} y$, where $N_{i}$ is the total number of protons in each beam. In the VdM scan, these integrals are evaluated by performing a scan in which the beam separation $\delta$ is varied in each plane, and the resulting rate $R(\delta)$ is measured. Factorizing into $x$ and $y$, we obtain $\int \rho_{x 1} \rho_{x 2} \mathrm{~d} x=R_{x}(0) / \int R_{x}(\delta) \mathrm{d} \delta$ for the $x$ direction, and similarly for the $y$ scan. Figure 1 shows an example of this scan procedure, as well as the beam positions throughout the overall 2017 program. The program includes four regular scan pairs (each consisting of one scan in each direction), as well as some special scans used to evaluate systematics.
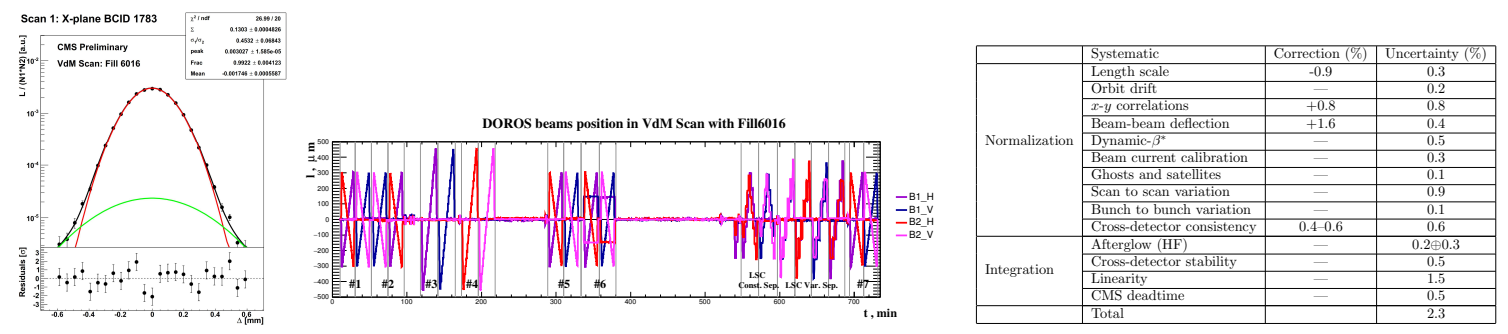

Figure 1: Left: Example of a rate vs. separation curve in a VdM scan, with resulting fit. Center: Positions of the two beams in $x$ and $y$ during the 2017 VdM scan program. Right: Summary of systematic uncertainties in the measurement. All figures are taken from Ref. [2].

Systematic uncertainties arise from the following. The VdM model assumes that the proton density functions are factorizable in $x$ and $y$; the effect of $x$ - $y$ correlations is measured with a special scan and a correction is applied. The length scale, which corrects for the difference between the nominal displacement of the beam and its actual displacement, is measured using a dedicated scan. Two beam-beam effects are considered, to account for the fact that when the beams are separated, electrostatic forces cause additional deflection and defocusing. Changes in the LHC orbit during the scan are measured using the beam position monitors. Additional uncertainties are assigned for scan-to-scan and bunch-to-bunch variation, consistency across the different CMS luminosity systems, and the measurement of the beam current. The overall uncertainties are summarized in the table in the right of Fig. 1. The total uncertainty is $2.3 \%$ for the overall 2017 run, and $1.7 \%$ for the special low-pileup LHC run. Full details of this measurement can be found in Ref. [2].

\section{References}

[1] CMS Collaboration, The CMS experiment at the CERN LHC, JINST 3 (2008) S08004, doi:10.1088/1748-0221/3/08/S08004.

[2] CMS Collaboration, CMS luminosity measurement for the 2017 data-taking period at $\sqrt{s}=13 \mathrm{TeV}$, CMS Physics Analysis Summary CMS-PAS-LUM-17-004, 2018. 